\title{
LITERATURWISSENSCHAFT
}

http://dx.doi.org/10.18778/2196-8403.2019.04

TORSTEN VOß

\section{,Ent-Spatialisierung'? oder: Das Scheitern der insu- laren Existenz in THEODOR STORMS Novelle Waldwinkel, mit Seitenblicken auf RAABE und STIFTER}

Der Beitrag beschäftigt sich mit dem Gelingen und Scheitern der insularischen Utopie (die aber nicht an die reale Insel gebunden ist wie so viele Entwürfe des Jahrhunderts, auf die allerdings knapp Bezug genommen wird) unter narratologischen Betrachtungen und Ansätzen aus dem Spatial Turn und bringt sie in Verbindung mit Auflösungstendenzen der romantischen, Waldeinsamkeit'. Zur Diskussion stehen dabei RAABES Das Odfeld (1889), ADALBERT STIFTERS Die Narrenburg (1844) und als garstiges und daher innovatorisches Gegenmodell vor allem THEODOR STORMS stark unterschätzte und wenig interpretierte Novelle Waldwinkel von 1874, die offen vorführt, wie Zeit und Geschichte selbst in die räumliche Utopie einbrechen und sie damit auf eine realistische Ebene transferieren.

"De-spatialization"? or the Fall of Insular Existence in THEODOR STORM's Waldwinkel in Reference to Prose by RAABE and SIFTER

The article deals with successful and unsuccessful constructions of the island utopia (which, however, are not bound to any real island, as in so many designs of the century, which are, however, briefly referred to), in terms of narrative issues and approaches from the Spatial Turn, and connects them with resolution tendencies of the so-called romantic "loneliness of the forest." Das Odfeld (1889) by RAABES and Die Narrenburg (1844) by ADALBERT STIFTER's are discussed, and in particular THEODOR STORM's greatly underrated and little-interpreted novel Waldwinkel (1874), as an unattractive and therefore innovative counter-model, which shows openly how time, and history itself, break into the spatial utopia, and thereby transfer it to a realistic level. 
„Odprzestrzennienie“ czyli upadek insularnych egzystencji w Waldwinkel TheOdora STORMa z odniesieniami do prozy RAABEgo i STIFTERA

Artykuł zajmuje się sukcesem i porażką utopii insularnej w kontekście refleksji narratologicznych oraz teorii spatial turn. Nie chodzi w tym przypadku o przedstawienie życia na wyspie, jak to czyni wiele projektów literackich dziewiętnastego wieku, do których krótko artykuł nawiązuje. Dyskutowany jest natomiast kryzys romantycznego toposu „Waldeinsamkeit" (osamotnienie w lesie). Analizie poddane zostały utwory Das Odfeld (1889) Wilhelma RaABego, Die Narrenburg (1844) AdAlBerTa StifTERA oraz niedoceniona i rzadko interpretowana nowela THEODORA STORMA Waldwinkel z 1874, będąca szorstkim i z tego powodu innowacyjnym przeciwieństwem do propozycji literackich Raabego i Stiftera. Tekst ten przedstawia, w jaki sposób czas i historia ingerują w utopię miejsca i przenoszą ją na płaszczyznę realistyczną.

\section{Vorab}

Reif für die Insel, selbst wenn sie gar nicht mehr im Meer, sondern im Wald liegt, sind auch noch in der realistischen Erzählliteratur des 19. Jahrhunderts einige Protagonist*innen, die in diesem Motiv bzw. Narrativ eine Alternative zu städtischen, merkantilistischen oder einfach auch nur pragmatisch-funktionalen Lebensformen und Denkauffassungen zu erreichen vermuten. Der Wunsch, diskursiven Zwängen zu entgehen, dominiert damit nicht nur den spätromantischen Künstlertypus, sondern auch die bürgerliche Existenzweise, wobei die Grenzen zwischen räumlicher Utopie und biedermeierlichem Idyll dabei teilweise fließend ausfallen können. Der Beitrag beschäftigt sich mit dem Gelingen und Scheitern der insularischen Utopie (die aber nicht an die reale Insel gebunden ist wie so viele Entwürfe des 18. Jahrhunderts, auf die allerdings knapp Bezug genommen wird) unter narratologischen Betrachtungen und Ansätzen aus dem Spatial Turn. Zur Diskussion stehen dabei WILHELM RAABES Das Odfeld (1889), ADALBERT STIFTERS Die Narrenburg (1844) und als garstiges und daher innovatorisches Gegenmodell vor allem THEODOR STORMS stark unterschätzte und wenig interpretierte Novelle Waldwinkel von 1874, die offen vorführt, wie die Zeit selbst in die räumliche Utopie einbricht und sie damit auf eine realistische Ebene transferiert.

\section{Die Insel als der andere Raum}

In Bezug auf Bernardin de Saint-Pierre und vor allem Chateaubriand lassen sich unter dem Kriterium des Unbekannten und Unentdeckten deren begeisterte Aufnahme, Bereisung und vor allem Schöpfung exotischer Ländereien und Inseln 
subsumieren, die außerhalb des europäischen Kontinents liegen, sei es die neue Welt Amerika oder ein mystisch und mythologisch verklärter Orient. Innerhalb der frühromantischen und vor allem von Friedrich Schlegel geprägten Kategorien des ,Interessanten “ und des ,Außergewöhnlichen“ scheinen die exotischen Inseln einen besonderen Raum einzunehmen. Sie symbolisieren einen Gegenentwurf zum Alltäglichen der lebensweltlichen Realität und scheinen deshalb als freie Imaginations- und Gestaltungsräume für das Selbstverständnis der romantischen Dichtungvon großer Bedeutung zu sein.

Auch noch Friedrich Schlegel, der sich dem restaurativen Metternichschen System angeschlossen hatte, feiert in seinen Wiener Vorlesungen über die $\mathrm{Ge}$ schichte der alten und neuen Literatur (1812) den Vorstoß ins Neue durch die französischen Frühromantiker, die auf diese Weise den formerstarrten Klassizismus und die permanente Verklärung der griechischen Philosophie hinter sich gelassen haben. Damals ,flüchteten Bernardin de St. Pierre und Chateaubriand ihre Einbildungskraft und Darstellung in die amerikanischen Wildnisse, wo sie nun von jenen unerbittlichen Tyrannen des französischen Mutterlandes, dem Aristoteles und Boileau nichts mehr fürchten durften“ (SCHLEGEL 1988:188). Vor dem Zugriff der Formenstrenge und der ,Imitation des Anciens' wird in die exotischen Gefilde ausgewichen. Die exotische Insel wird nicht nur zur sozialen oder erotischen Utopie, sondern auch zur Spatialisierung eines neuen, sich ganz und gar der freien Imagination überlassenden Kunstverständnisses, zum räumlich fassbaren Narrativ einer neuen Mythologie. Die Verräumlichung einer Kunst- und Sozialutopie postuliert noch auf idealistische Weise die Möglichkeit zur Konstituierung eines Fluchtraums. Es scheint möglich zu sein, Kunst- und Lebenskonzepte visualisierbar zu machen. Doch das ändert sich. Die exotischen Hütten werden nicht nur durch die typischen Korrespondenzverhältnisse mit der Außenwelt in ihrer idyllischen Funktion relativiert, ihre Bewohner*innen tragen selbst den Bezug zum Außen a priori in sich, was jegliche Verklärung der Isolation ad absurdum führt, auch schon in Bernardin de Saint-Pierres Paul et Virginie und dem René Chateaubriands (1962), welcher in seiner Sehnsucht nach Entgrenzung den Tod auf dem Schlachtfeld findet (vgl. MALER 1978). Eine Ausnahme könnte jedoch der andere Hütten-Roman Saint-Pierres bieten, also La chaumière indienne von 1791. Aufgrund der damaligen Bekanntheit des Rousseau-Schülers, die vielleicht sogar bis nach Husum, Wien und Wesselburen durchgedrungen ist, könnte die dort entworfene insularische Existenz der vorbildhafte Intertext für STORMS pessimistische Narration sein, zumal auch hier schon an die Stelle des Meeres der Wald getreten ist. Die Familie eines 
ausgestoßenen Parias bildet dort mit Hund und Katze eine kleine Gemeinschaft. In der Abgeschiedenheit des Waldes suchen die Liebenden, also der Paria und eine Brahminenwitwe, die der Einsiedler vor der Verbrennung bewahrt und aufnimmt, die solitude à deux, die nur vom Feuer der Liebe erfüllte Natur, wie sie bereits von Rousseau entworfen wurde. Oder wie es Anselm Maler auf den Punkt bringt: „In der Einsamkeit sind sie einander alles. Unbelastet vom Wissen um die vergangenen Dinge leben beide in geschichtslosem Glück“ (MALER 1978:193).

Diese Entdiskursivierung scheint auch das sich bereits selbst überlebende Ziel des ehemaligen Revolutionärs Richard zu sein, der sich mit seinem Mündel Franziska in den von ihm besorgten Waldwinkel in THEODOR STORMS gleichnamigen Erzähltext zurückziehen möchte. „Der umschlossene Naturschauplatz, ein Raum geschichtsloser Privatheit; der konzentrisch errichtete locus amoenus, Reservat intimer Geselligkeit, sie bilden den Aufenthalt der von der Großen Welt Abgewandten seit dem späten Rokoko“ (MALER 1978:191), wie es Maler formuliert - und wohl auch noch im industriellen und bürgerlichen Zeitalter des späten 19. Jahrhunderts. Doch was ist dabei Utopie, Lebensweise oder nur noch Zitat fernab jeglicher Realisierung im Waldwinkel von Richard und Franziska? Wovon werden die Bewohner*innen der Klause geleitet? Wodurch erfährt der Hüttenentwurf Bernardin de Saint-Pierres seine Pervertierung bzw. seine desillusionierende Entmythologisierung, so dass die Insel am Ende nur noch als „Symbol der existentiellen und unüberwindbaren Einsamkeit und Selbstbegegnung" (BROSER 2012:200) in Anspruch genommen werden kann, wie es Patricia Broser für das moderne Inselverständnis postuliert hat (vgl. auch die ältere Monographie v. BRUNNER 1967)? Eine Problematik utopischer Habitate und Existenzweisen bezüglich der eigenen Lebensgestaltung hat Ralph Rainer Wuthenow bereits in den achtziger Jahren herausgearbeitet: Für viele Entdeckungsreisende gilt, dass ,die Erwartungen der Reisenden nicht selten schon quasi utopisch verformt sind" (WUTHENOW 1985:324). Der fiktive Entwurf der Insel ist die Schablone, an welcher die Realität gemessen und vielleicht sogar ausgestaltet wird. Insofern sollte eine Verknüpfung mit narrativen Verfahren nicht überraschen.

\section{Die Insel als Kasten? THEODOR STORMS Waldwinkel in der Kritik}

Auch wenn STORMS Novelle von 1874 keine konkreten Hinweise über eine mögliche Bernardin de Saint-Pierre-Lektüre Richards liefert, wird er von einer Vision geleitet, die es architektonisch, sozial und habituell umzusetzen gilt. Das 
heißt: Er übernimmt wie die mitunter enttäuschten Reisenden des späten 18. Jahrhunderts ein Modell, an dem sich die reale Lebensführung wie an einem Entwurf auszurichten hat. ${ }^{1}$ Das Ziel ist auch für STORMS Waldbewohner*innen immer noch ,ein Zustand, wie die europäische Gegenwart ihn verweigert, [...] Wunsch und Traum und Denken drängen über das bestehende Wirkliche hinaus“ (WUTHENOW 1985:323) oder schreiben sich in eben dieses Wirkliche mehr oder weniger erfolgreich hinein, um das Titelgefüge, also den Waldwinkel zu konzipieren - und das vor dem Hintergrund einer modernen Industrie- und Kapitalgesellschaft. Es verwundert also nicht, dass STORMS Umfeld dem Text bisweilen irritiert gegenüberstand.

Auf die Novelle Waldwinkel von 1874, die STORMS mittlerer Schaffensperiode zuzurechnen ist, hat die zeitgenössische Kritik alles andere als begeistert reagiert, vor allem in Gestalt der Dichterkollegen Turgenjew und Fontane. Der erste schreibt am 27. November 1874 an den Maler und Kritiker Ludwig Pietsch: ${ }^{2}$

Ich habe mich über den Waldwinkel hergestürzt ... und ... und muß bekennen, daß ich Ihren gewöhnlichen Scharfsinn vermißt habe. - Mein Freund, dieses Stormsche Produkt ist schwach - ,Ich schwör es bei unserer Liebe“ wie Ottavio im Don Juan singt. - Alles ist hart, unmotiviert - man wird von keiner der drei Figuren gewonnen, selbst vom Hund nicht, der auch etwas literarisch aussieht, und die Poesie wird wie Butter aufgeschmiert. Vielleicht irre ich - aber vielleicht auch Sie. (TURGENJEW 1968:95)

Einige der wichtigsten Faktoren novellistischen Geschehens, also Dynamik der Handlung, klar erkennbare Leitmotive und ein wesentlicher Konflikt, sind

1 Genau das wäre nach Wuthenow problematisch. Das wahre Arkadien existiert unabhängig von Schemata und Vorgaben. Mit Blick auf die Tahiti-Erlebnisse der Weltreisenden Georg Forster und Bougainville kommt er zu der Beobachtung: „Das glückliche Dasein war hier nicht Erinnerung oder Traum in die Zukunft hinein, sondernganz gegenwärtiges Dasein“(WUTHENOW 1985:229). Es fällt also aus der Zeit heraus und wird geschichtslos, kann aber gerade deshalb nicht dauerhaft von den Seeleuten als Existenzweise gelebt werden. Denn diese sind Teil der Geschichte und der Kontinuität, versinnbildlicht auch in der Reise. Dadurch bleibt der Inselraum Utopie, von der nur gekostet werden darf. Das stellt übrigens eine große Disparität zu STORMS Erzählung von 1874 dar. Richard will seiner Utopie, dem Waldwinkel, dauerhaft teilhaft bleiben. Es kommt zur Stagnation, der sich die junge Franziska letztendlich verweigern wird, zumal die Realisierung der Utopie das utopische (und damit auch kritische) Denken - gemäß dem Prinzip Hoffnung Ernst Blochs - beenden würde.

2 Dieser hatte STORMS neues Werk am 07. Oktober 1874 emphatisch begrüßt und später auch gegenüber Iwan Turgenjew, der selbst mit STORM brieflich korrespondierte, empfohlen. 
folglich nach Turgenjew beim Waldwinkel nicht erfüllt. Auch wird hier, im Gegensatz zu den maritimen Inselutopien und zu den romantischen Waldeinsamkeiten, nicht gereist. Der Ort wird errichtet und alles bleibt daraufhin merkwürdig statisch. Ebenso kann er auch keine Verbindung zu den handelnden Figuren herstellen, also zum Besitzer des Waldwinkels Richard, der jungen Franziska und dem jungen Försterburschen, welcher schließlich die junge Frau dem Waldwinkel abspenstig macht. Auch das würde gegen STORMS eigene Novellentheorie verstoßen, welche in der anthropologischen Relevanz der Schicksale seiner Figuren auch den eigentlichen Anreiz für das Interesse des Publikums sieht. Theodor Fontane geht sogar noch härter mit dem ansonsten so geschätzten Kollegen ins Gericht. In seinen unvollendet gebliebenen Erinnerungen an Theodor Storm fasst er zusammen:

Was Landschaft, Lokalschilderung (das alte Haus Waldwinkel), Stimmung, Schwüle, Bibber angeht, so hat er hier sein Äußerstes geleistet, weit über Immensee hinaus. Das Ganze ist aber der reine Quatsch, unwahr, eklig, raffiniert. Daß ein Mann von 48 eine junge Person von 18 mit in seinen Waldwinkel nimmt, um hier wie drei Auerhähne zu balzen, mag geschehen und auch novellistisch behandelt werden. Wenn sich der rechte Mann dazu findet, so kann es famos, unter Umständen großartig sein, - hier aber wirkt alles dünn, kläglich, impotent, im höchsten Maße unerquicklich. Nicht bloß das Liebesverhältnis, mehr noch das, was drum herum ist, ist aufs äußerste raffiniert. Das Ganze ein wahres Musterstück, wie man's nicht machen, wie Kunst nicht sein soll. (FONTANE, zit. nach STORM 1987:864)

Während Turgenjews Vorwürfe sich eher auf angebliche stilistische Mängel beziehen, argumentiert der große Geschichtenerzähler und Dialogliebhaber Theodor Fontane inhaltlich, bisweilen sogar auf der moralischen Ebene.

$\mathrm{Zu}$ konstruiert kommt Fontane anscheinend der von jüngeren Frauen angezogene Richard vor, der am Ende der Novelle von seiner Geliebten aufgrund eines forschen und frischen Nebenbuhlers verlassen und allein in seiner Waldinsel zurückgelassen wird und damit einen Stoff kultiviert, der nach Fontane der hohen Literatur nicht angemessen ist. Auch die damalige Leserschaft warf dem Novellisten STORM einen unüberbietbaren Pessimismus hinsichtlich des Finales der Erzählung vor. In einem Brief an Paul Heyse vom 25. November 1874 zitiert STORM das Urteil einer jungen Leserin, der Baronin Hermione von Preuschen: „Die Geschichte ist nicht melancholisch, sie ist pessimistisch!“” (FONTANE, zit. nach STORM 1987:865) Recht hat sie und auch FONTANE ist mit seiner vernichtenden Wertung von der Wahrheit nicht weit entfernt. Denn sowohl einem Raumals auch einem Sozialkonzept wird eine radikale Absage durch den Erzähler erteilt bzw. beides erfährt seine Karikatur. 
Allerdings wäre es verfehlt, der Novelle aufgrund einer Stagnation des Plots und mangelnder Emphase gegenüber den Akteuren Qualität abzusprechen, denn die Narration muss ihre Dynamik zwangsläufig aufgeben, das Ende muss hoffnungslos sein und der alternde Richard muss an seinen Ansprüchen, Zeit und Geschichte aus der Klause zu verbannen, scheitern, um zur tragischen Figur im Sinne von STORMS Novellenpoetik zu werden. Nur so kann sein Schicksal berühren und als anthropologisch relevante Krise verstanden werden, hier versinnbildlicht am Versagen einer räumlichen Utopie, die zugleich das Narrativ in seiner Stagnation bestimmt, ja das tatsächlich das Narrativ selbst und nicht nur Teil der Diegese ist, wie beispielsweise die Figuren und deren Handlungen (dazu mehr in MARTíNEZ / SCHEFFEL 2016:153-158). Vielmehr geht alles von der Raumvorstellung selbst aus. Sie greift in die Entwicklung des Plots und der in ihm agierenden Figuren ein. ${ }^{3}$ Denn anhand der spatialen Projektion Richards und der in sie inkludierten Franziska konstruiert STORM den Dualismus von Außen und Innen, von Raum und Zeit.

Diesen wird später Jurij Lotman als semiotisches und strukturierendes Grundprinzip von Raumerzählungen unter dem Aspekt der Grenze bestimmen (vgl. LOTMAN 1974:200-271). Grenzsetzungen und Grenzüberschreitungen sind auch die Regularien, mit denen die Protagonisten Richard und Franziska ihr Habitat zu gestalten versuchen. Richard konstruiert den Waldwinkel. Franziska wird ihn durchbrechen und damit den Konstrukteur der Grenze zurücklassen, da beide Grenzvorstellungen nicht miteinander zu fusionieren sind. Man muss daher mit Michael C. Frank feststellen: „Die räumlichen Relationen zwischen Objekten werden zur Basis für die Darstellung nicht-räumlicher Relationen, wenn zum Beispiel ,hoch/niedrig' mit ,gut/schlecht' oder ,nah/fern' mit ,eigen/fremd“ gleichgesetzt wird“"(FRANK 2012:221). Sie sind also auf figurale Konstellationen wie Richard und Franziska zu übertragen, da jede Seite eine Relation zur spatialen Titelfigur bei STORM eingeht. Das heißt: „Räumliche Strukturen in der Literatur vermitteln demnach einen Einblick in die Art und Weise, wie die dazugehörige Kultur die, Welt' konstruiert“" (FRANK 2012:221). Daraus lassen sich Rückschlüsse auf die jeweiligen Konstrukteure ziehen, welche

3 Noch vor allen kulturwissenschaftlichen Modeströmungen im Sinne des Spatial Turn sind Konstellationen dieser Art anhand von STORMS Waldwinkel bereits herausgearbeitet worden durch den originellen Aufsatz von JOSEF DE CORT (1971:321-340, bes. 338f.). Eine Kompilation aktueller raumwissenschaftlicher Ansätze (und deren Vorbereitung durch Semiotik und Strukturalismus) findet sich bei HaLlet / NEUMANN (2009). 
die innere und die äußere Räumlichkeit ebenso verkörpern wie daran gekoppelte Lebensalter und einen disparitären Umgang mit der Zeit.

Immerhin thematisiert STORM über diese Raumvorstellung auch zwei der größten Ängste, welche das menschliche Dasein zutiefst verfinstern, die vor der Einsamkeit oder - noch schlimmer - die vor dem ,Zurückgelassenwerden' und die vor dem Alter. Beides kulminiert in der Figur des Insulaners Richards, welcher sich in leidenschaftlicher und einengender Liebe zu der jungen Franziska verfängt. Er plant mit ihr eine gemeinsame Existenz im von ihm erworbenen und einsam gelegenen Waldwinkel, also eine Art insularisches Dasein, welches nur aus der Liebe zwischen Partner und Partnerin inmitten einer idyllischen Naturkulisse belebt werden soll und auf Kontakte mit dem externen Soziotop zu verzichten hat. In mythologischer Hinsicht versteckt sich dahinter, wie bei den maritimen Insel-Utopien à la Tahiti, ,der Wunsch, die Folgen der Austreibung aus dem Paradies wieder rückgängig zu machen“"(WUTHENOW 1985:324), wie es WUTHENOW ausdrückt. Das vorreflexive und ahistorische Leben aus sich selbst heraus ist immer noch der versteckte Intertext, der den enttäuschten Ex-Revolutionär Richard motiviert, als er sich mit seinem Mündel den Waldwinkel einrichten will. Darin unterscheidet er sich stark von den Inselkonstrukteuren anderer Autoren im 19. Jahrhundert. Durch den Modus des Zwangs und des Ausbleibens einer gemeinsamen Konstruktion der Waldinsel ist das Scheitern der spatialen Utopie als romantisches Ideal bereits ihrer Schöpfung inkludiert. Das alternative Lebenskonzept Richards birgt den Tod in sich, was für viele Beziehungsmodelle im novellistischen Werk THEODOR STORMS zu veranschlagen ist. Marianne Wünsch hat herausgearbeitet, dass STORMS Figuren mitunter schon im „Zustand eines metaphorischen Todes zu Lebzeiten“ (WÜNSCH 1999:6) gefangen sind. Der Rückzug in die temporär stagnierende Enge des Waldwinkels vollzieht diesen von Wünsch prognostizierten Tod symbolisch-allegorisch, macht ihn zum anschaulichen Narrativ. Das deckt sich auch mit der Figur Richards, dem zurückgebliebenen und seiner Zeit entzogenen Ex-Revolutionär, an dem die Geschichte vorbeigelaufen ist. Er gehört deshalb zu all den ,im Prozess der Liebesfindung, der Ehegründung, der Zeugung oder Erziehung gescheiterten Figuren“ (KÜNG 2015:23), die nach Peter Küngs Einschätzung immer wieder die Novellen STORMS mitbestimmen. 


\section{Wie man eine Insel anlegen könnte: RAABE und STIFTER}

WILHELM RAABES gelehrter Magister Bucchius von Amelungsborn in Das Odfeld (1889) wird von ganz anderen Motiven geleitet. Sowohl die eigene klösterliche Existenz in der Schreibstube als auch die für seine auserwählte Fluchtgemeinschaft requirierte Höhle sind Schutzräume eines humanistischen und gelehrten Geistes. Der kauzige Pädagoge wird als ein „vollkommen passiver Held“ (RAABE 1985:21) inszeniert. Das ist spezifisch für RAABES Figuren und Erzählweise im Odfeld und darüber hinaus: Passivität (und bei Bucchius mitunter auch Resignation angesichts der Korrumpierbarkeit der Menschen) unterbindet Aktionismus und direktes Eingreifen von Seiten des Charakters. ${ }^{4}$ Auf der narratologischen Ebene impliziert es das Verhindern einer geradlinig fortbestehenden, sich rasch entwickelnden Handlung und setzt dagegen Innenbetrachtungen der Figur und Momente des Verweilens und Abschweifens (vgl. RITTERSON 1976:107-132). ${ }^{5}$ So resümiert denn auch Walter Killy: „Es ist dies viel Ancienneität für einen armen Teufel, den die praktische Behörde zum Schuldienst untauglich befand, und viel Geschichte für die Schilderung der vierundzwanzig Stunden, die das Buch darstellt" (KILLY 1963:130). Durch ihre allegorische Inkarnation ausgedrückt wird die Ausdehnung bis hin zur Langeweile zum entscheidenden Stilelement bei WILHELM RAABE, sowohl als Modus der Figuren- und Raumkonzeption als auch des Erzählens. Die wechselseitigen Beziehungen zwischen Personalkonstruktion und narrativem Verlauf sollen nicht nur an Textbeispielen verdeutlicht, sondern auch mit Blick auf RAABE als Autor des Realismus behandelt werden. Charakterzeichnung und Orts- und Zeitgestaltung sind in RAABES Das Odfeld als konstitutives Bedingungsverhältnis zu rezipieren. ${ }^{6}$ Die Eigenschaften des Magisters Bucchius von Amelungsborn drücken sich nicht nur

4 Genaueres über Verhalten und Selbstverständnis des Magisters Bucchius erfährt man bei OPPERMANN (1967:31-50).

5 Der Aufenthalt in klösterlicher Schreibstube und Höhle sind die räumlichen Entsprechungen des Verweilens und Absonderns.

6 Meine Ausführungen dokumentieren bereits, dass mein Erkenntnisinteresse sich auf räumliche Konstruktionsprinzipien und figurative Inszenierungsverfahren (Magister Bucchius) ästhetischer, die Lektüre absichtlich erschwerender, Zeitausdehnung zwecks Wirklichkeitserfassung bezieht und eher peripher auf die starke Symbolik von RAABES spätem Roman rekurriert. Eine Darlegung von Das Odfeld und das über ihm sich vollziehende geheimnisvolle Prodigium / Praesagium der 
über seine Handlungsweise, sondern auch in der Beurteilung durch den Erzähler aus. Dort erscheint der Gelehrte als Relikt der Vergangenheit, einer Zeit außerhalb der Zeit, als etwas Überflüssiges, wenn es über seinen Verbleib im alten Kloster heißt:

Und doch - doch hatten sie ihn bei ihrem Abzuge nicht mit sich genommennach Holzminden, in die neue gelehrte Herrlichkeit, sondern ihn zurückgelassen am alten Ort, allein in den leeren Auditorien und Dormitorien, vor den jetzt so gespenstischen Subsellien und in seiner Zisterziensermönchszelle über dem Hooptale als das unnützeste, verbrauchteste, überflüssigste Stück ihres Hausrats! (RAABE 1985:21)

Hierin finden sich, wie noch zu demonstrieren sein wird, die entscheidenden Attribute der eigentlichen Erzählstrategie und Raumgestaltung. Bereits an der Begründung für das Zurücklassen von Bucchius lässt sich so manches ablesen. Sie liegt im Aktivismus begründet, der sich aus den Wirrnissen des Siebenjährigen Krieges ergibt, womit RAABE gleichzeitig auch auf eine Dynamisierung von Existenz und Kommunikation, auf ein Zeitalter der wachsenden Mobilität anspielt. Passivität ist für den frühmodernen Menschen undenkbar, und so wird der Magister von seiner Umwelt quasi zur Insel erklärt. Daraus ergibt sich auch die Zäsur zwischen dem Personal der Klosterschule und dem Magister Bucchius als eine Art Relikt:

Man hatte einfach eben wieder einmal nicht gewußt, was man tat, - wer kann denn aber im Tumult des Lebens und eines Hauswechsels sich recht auf alles besinnen? [...] Dreißig Reichstaler des Jahres ließen sie ihm und die Zelle des Bruders Philemon bis zu seinem Lebensende. (RAABE 1985:21f.) ${ }^{7}$

Bucchius ist die insularische Gegenfigur zur beginnenden und ihn umspülenden Massengesellschaft, und dennoch liegt in seiner Passivität die Rettung einer kleinen Gruppe von Schülern, Angestellten und einer jungen Aristokratin

Rabenschlacht (vgl. RAABE 1985:28-34) als Vorausdeutung für kriegerische Auseinandersetzungen im Siebenjährigen Krieg und deren Auswirkungen auf den Magister Bucchius und seine unmittelbare Umgebung ist bereits von anderer Seite überzeugend geleistet worden, unter anderem durch den Aufsatz von HEINRICH DETERING (1984:87-98).

7 Diese Hinweise markieren, dass Bucchius mit seiner Zelle einen Raum gefunden hat, welcher dem Ablauf der Zeiten entzogen ist. Er ist praktisch ,stillgelegt " und der Erzähler versäumt es auch nicht zu erwähnen, dass passive Menschen die Möglichkeit haben, ,es dann und wann vor allen andern Menschenkindern zu einem hohen Alter zu bringen“"(RAABE 1985:21). Langlebigkeit steht also in einem untrennbaren Zusammenhang mit Passivität, womit wiederum auf die ausbleibenden Handlungselemente verwiesen wird, die dem Roman seine künstliche Länge verleihen und durch den Magister Bucchius ihre visualisierende Inkarnation erfahren. 
begründet, welche er aus dem von feindlichen Truppen heimgesuchtem Kloster wie eine Art Noah in seine Arche in Gestalt einer Höhle führt. Diese ist ebenso wie die Klosterzelle als Refugium vor der Kontingenz des Daseins zu betrachten. Außerhalb der Höhle herrscht das Chaos des Krieges, welches ja schon in der Rabenschlacht über dem Odfeld sein Praesagium gefunden hat. Die Höhle widersteht jedoch dem durch den Krieg symbolisierten diskursiven Wandel ebenso wie der in Amelungsborn zurückgelassene Insulaner Magister Bucchius, der auf betont langsamere Töne setzt. RAABE besetzt diese passive und stets abwartende Figur in ihrer Rolle als Lebensretter positiv und verteidigt damit auch seinen Erzählstil einer zeitlichen Zerdehnung, der sich in permanenten Abschweifungen und historischen Exkursen ergeht, anstatt sich der gegenwärtig dominierenden Sprache der Beschleunigung anzupassen. ${ }^{8}$ Durch die zahlreichen Verweise und Subthemen, die der Text enthält, gleicht er auch dem Archiv des Magisters Bucchius, seinem Sammelsurium an Artefakten, dessen Reichhaltigkeit nur mit Geduld und erhöhter Aufmerksamkeit erfasst werden kann. Damit liefert der Autor auch eine ironische Anspielung auf die Schwierigkeiten, mit denen man sich durch RAABES Texte kämpfen muss, deren Verfasser sich in der Gestalt des Magisters auch als poeta doctus präsentiert, ,der freilich, um begriffen zu werden, auch eines gebildeten Publikums bedarf" (KILLY 1963:135), wie es Walter Killy betont. Ein anderer Rezipient*innenkreis, der an schnellere Lektüren gewohnt ist und mit den zahlreichen Anspielungen auf die Geschichte der abendländischen Kultur, sei es die christliche Passion oder die antike Philosophie der Stoiker, kaum etwas anzufangen weiß, wird durch jenes Aufhalten des Handlungsflusses eher frustriert. Ähnlich geht es ja dem jungen Heißsporn und Schulversager Thedel von Münchhausen, welcher mit der Passivität und Seelenruhe des Magisters kaum etwas anzufangen weiß und außerhalb der Insel-Höhle prompt den Tod findet. Dass die Langsamkeit des Magisters - im Gegensatz zu Richard in STORMS Waldwinkel - auch ein ethisches Potential in sich trägt, wird durch die mythologische Konnotierung seines insularischen Refugiums, der Höhle, ausgesagt. Das erkennt auch Killy:

8 Das wird auch darin ersichtlich, dass sich die ,Höhlenbewohner' Spukgeschichten und Ähnliches erzählen, im Medium der Narration also ganz bewusst Ablenkung vom hektischen Treiben der Gegenwart suchen (vgl. RAABE 1985:162). Zugleich wiederholt RAABE mit dieser Ablenkungs- und Beschäftigungsstrategie auf intertextuell anspielungsreiche Weise das anthropologische Bedürfnis nach dem Erzählen, wie es sich auch in Boccachios Il Decamerone und in Goethes Unterhaltungen deutscher Ausgewanderten angesichts der Isolation von der Umwelt findet. 
Die Funktion der Höhle, auf die es Raabe vorzüglich ankommt, wird erst ermöglicht, indem der Ort seine einmalige Position verliert. Aus der Höhle am 5. November 1761 wird eine Fluchtstätte zu aller Zeit; der Dolomit des Ith unweit der Weser wird zum Gebirge Judäas, der deutsche Wald zur thebaischen Wüste: überall und immer hat der Mensch sich eine Zuflucht suchen und dem Rat des Magisters folgen müssen: ,...stehet oder sitzet und gewöhnet eure Augen an die Finsternis‘ . (KILLY 1963:134f.)

Dass diese insularischen Fluchtpunkte am Ende nicht tragen, liegt jedoch, anders als bei STORMS Richard-Figur, nicht an der Verfassung des Protagonisten, sondern seiner Schutzbefohlenen wie Thedel und an der Unmöglichkeit, die moderne Massengesellschaft hinter Archiven, Lektüren und biblischen Raumphantasien auszublenden. Thedel ist bereits ein Teil von ihr, er ist dem Schutzraum entzogen und infiziert damit den ou-topos, der aus ähnlich lauteren Motiven errichtet wurde wie schon ADALBERT STIFTERS Narrenburg. Außerhalb der Enklave findet er dann auch schnell den kriegsbedingten Tod.

War es in STIFTERS dreißig Jahre zuvor entstandener Erzählung Die Narrenburg von 1842 bzw. 1844 wie in Bernardin de Saint-Pierres ,indischer Hütte ' den Protagonist*innen Anna und Heinrich im gemeinsam errichteten und wieder zum Leben erweckten ou-topos noch möglich, ${ }^{9}$ fernab von aller Zivilisation und nur von ihrer Liebe und der Luft zu leben, ist das Richard und Franziska nicht mehr gestattet. ${ }^{10}$ Das resultiert nicht nur aus dem großen Altersunterschied, der bei STIFTERS Charakteren nicht vorhanden ist, sondern auch daraus, dass hier die Liebe und das Begehren Richards viel materialistischer und damit auch düsterer (und zum Scheitern verurteilt) wirkt als bei STIFTER. Bei Letzterem ergibt sich aus dem letzten Nachfahr der Scharnasts und seiner Gefährtin eine neue Vitalität, die aus den maroden Mechanismen des Adelsgeschlechts auszubrechen vermag. Die rousseauistische Unschuld und die daraus resultierende gemeinsame - auch lebenspraktische und handwerkliche - Kreativität der Bewohner der Narrenburg (vgl. SCHRÖDER 2012:30-48 u. 69-78) weicht bei

Als Traumprojekt wird es inszeniert durch die Kinder René und Florence in Robert Brasillachs Comme le temps passe aus dem Jahr 1937. Sie leben und gestalten sich darin ihre präzivilisatorische Inselutopie, welche aber eher den Kulturpessimismus des später zu den Nationalsozialisten übergelaufenen Romanciers und Kollaborateurs offenbart und an die Stelle einer als unzureichend empfundenen Moderne den antikisierenden Mythos mit deutlicher Anlehnung an Rokoko-Ästhetiken setzt (vgl. BRASILLACH 1953).

10 Diese versöhnliche Gegenmodulation bei STIFTER behauptet vor allem JUTTA MÜLLER-TAMm (2007:561-574). 
STORM einer verzweifelten Leidenschaft, ja einer amour fou. In Opposition dazu bricht Heinrich nach Ansicht von Saskia Haag mit aller Genealogie, die sich hinter den Verfahren des Abschließens verbirgt: „Er ersetzt die lineare, chronologische Zeitrechnung durch eine heilsgeschichtliche Typologie, die eine Kontinuität über Brüche hinweg ermöglicht und in der Heinrich in messianischer Gestalt ,zurückgekehrt" ist, um das Geschlecht der Scharnasts zu erlösen“ (HAAG 2012:75). Die Erlösung liegt - im Gegensatz zu STORMS manifest gewordener Waldbehausung - gerade in der Aufhebung von räumlicher und existentieller Stagnation begründet. Ein neues Konzept gemeinsamer Selbst- und damit verbundener Raumrealisierung tritt an die Stelle der Scharnastschen Verfalls-Genealogie, als Heinrich und Anna die Narrenburg betreten und in Teamarbeit revitalisieren. Auch Karl Ernst Laage erkennt vollkommen korrekt, dass

auch das Verhältnis der Liebesleute zueinander grundverschieden ist: bei Stifter ist es von Scheu und Scham bestimmt, bei Storm wird es als ,schwül' gekennzeichnet. Entsprechend ist der Schluß der Stormschen Novelle ein krasses Gegenbild der Stifterschen: Stifters Heinrich gelingt es, mit Anna zusammen die alte Narrenburg zu neuem Leben zu erwecken; Storms Narrenkasten aber wird ausgekehrt und abgeschlossen (S. 278, 22). Damit erweist sich die biedermeierliche Idylle des Anfangs in Storms Novelle als Illusion. Es ist, als habe der Husumer Dichter sagen wollen, daß Stiftersche Inselexistenzen in seiner Zeit nicht mehr möglich seien (LAAGE 1987:867f.),

da sie von der Zeit und der Geschichte selbst überholt zu werden scheinen. Andere Diskurse, zum Beispiel die Stadt oder hier bei THEODOR STORM die Jugend, brechen in die insularische Lebens- und Sozialform ein und relativieren diese. Die Brisanz der Schlusspassage liegt darin begründet, dass auch das Inseldasein sich auf Gemeinschaft oder besser gesagt: Zweisamkeit gründet, da dessen Erfolg als Raumkonzept vom erfüllten Liebesglück Richards abhängig ist. Ist dieses erst einmal gescheitert, wird der locus amoenus zum locus terribilis. ${ }^{11}$

\section{Flucht von der Insel und die Pervertierung des Waldwinkels}

Der abgeschiedene Ort steht bei STORM für den Zustand der Zurückgelassenheit als eine negative Variante der Selbstfindung, denn er wurde ja nur unter den Bedingungen der Zweisamkeit und der erfüllten Liebe errichtet. Fällt diese

11 Man stelle sich nur einmal vor, Daniel Defoes Robinson Crusoe hätte seinen Freitag auf der Insel niemals gefunden. Er wäre gewiss verrückt geworden und aus den Kolonialprojekten wäre ohne Kommunikation kaum etwas realisiert worden. 
restlos weg, verliert auch der verklärte Ort seine Legitimation. Von nun an steht er nur noch für das Scheitern und die absolute Einsamkeit, die schlussendlich auf den Tod verweist. Insofern ist die eigentliche tragische Figur der Ort Waldwinkel selbst, weil er die mit ihm verbundenen Hoffnungen, Sehnsüchte und Erwartungen nicht erfüllen kann, ja nach Franziskas Weggang nur noch für das genaue Gegenteil steht. Um diese niederschmetternde und in der Tat pessimistische Erkenntnis zu visualisieren, ${ }^{12}$ muss der ehemalige Revolutionär Richard an der hereinbrechenden Zeit scheitern, muss die Novelle voller Antizipationen des tristen Endes stecken, so auch durch das mehrmalige Auftreten des jungen Försters, einer Figuration des Dritten, die ja stets dem Raumkonzept des Insularischen gegenüber als inkompatibel ausgerichtet ist. Diese Einflussnahmen sind zu berücksichtigen, wenn man wie Georg Bollenbeck die These aufstellt, Storm entwerfe „keine erotisch-bukolische Geschichte“ (BOLLENBECK 1988:296). Der Waldwinkel ist kein Kythera, in das man sich nach der Manier Antoine Watteaus auf pittoreske Art einschiffen kann. Natur und Mensch sind in der Postromantik einander entfremdet, da der Waldwinkel allein dem Begehren Richards gehorcht und damit von Anfang an einer Pragmatik subsumiert ist, was jedem Verständnis von Bukolik und Arkadien zuwiderläuft. Nach Bollenbeck zeigt sich das auch in den veränderten Naturbeschreibungen durch den auktorialen Erzähler, denn ,die Flucht aus der zivilisierten Gesellschaft in die unberührte Natur erreicht nicht ihr Ziel, denn auch die Natur verweigert sich als Refugium. Vielmehr geht von ihr eine bedrohliche Unruhe aus" (BOLLENBECK 1988:296). ${ }^{13}$

Bukolische Insel-Idyllen scheitern nicht nur an der wenig amönisch anmutenden Natur, sondern auch daran, dass soziale Belange wie Franziskas Bedürfnis nach pekuniärer Sicherheit stärker ausfallen als der Wunsch nach Äquivalenz

12 Damit geht Theodor Storm meines Erachtens weit über eine melancholische Stimmungsnovelle wie Immensee (1849) hinaus und nähert sich wieder stark seinen Liebes- und Abschiedsgedichten, ja sogar dem nihilistischen Tenor von Geh nicht hinein (1879) an (vgl. VОß 2010:81-96).

13 Was Emil Kuh gegenüber Theodor Storm in einem Brief vom 06. Januar 1875 noch stark kritisierte, also eine breite und dunkle Landschaftsausmalung, wurde von diesem stark verteidigt als ein Stilmittel, ,um dem Ganzen die Stimmung der Abgeschiedenheit zu geben“ bzw. den „Eindruck der Abgeschiedenheit von den Menschen u. des gleichsam Verlorenseins in die Natureinsamkeit" hervorzubringen (STORM 1987:864f.). Das leuchtet insofern ein, als der Wald hier nun an die Stelle des Meeres getreten ist und eine ähnlich dichotomische Funktion erfüllt. Er soll in einer Art Wiederbelebung Tieckscher Waldeinsamkeit die Zivilisation ausblenden. Was jedoch bei Tieck ein poetologisches Programm visualisierte, wird hier zur Karikatur. 
zwischen Lebensweise und Natur. Das mit der Inselkonzeption in unmittelbaren Zusammenhang stehende romantische Konzept der Waldeinsamkeit erfährt hier seine Relativierung, was auch auf die Veränderungen eines literarischen Topos verweist, der sich selbst überlebt hat und zum reinen Zitat wird. Bollenbeck verweist ebenfalls auf den Bruch mit der literarischen Tradition, wenn er nüchtern erkennt: „Nun kann der Rückzug aus der Gesellschaft, die Flucht vor ihren Zumutungen und Konventionen in die ,Waldeinsamkeit' nach Tieck, Eichendorff und STIFTER eher als konventionelles, trivialliterarisch verbrauchtes Motiv gelten“" (BOLLENBECK 1988:296). ${ }^{14}$

Wenn STORM schon einmal die insularische Existenzweise als Liebes- und als soziales Glück perspektiviert, dann geschieht das lediglich unter dem Vorzeichen des Traumes wie in der frühen und mythologisch angehauchten Prosaskizze Celeste (1840). Das Haus im Waldwinkel steht nicht für ein dauerhaftes Glück und schließt sich an die Tradition einer schwarzen und skeptischen Aufklärung an: Bereits Bernardin de Saint-Pierres Rousseau-Roman Paul et Virginie (1788) attestiert, dass der Mikrokosmos der Insel zusammenbricht und die mit ihm verbundenen Glücksvorstellungen sich auf dialektische Weise in ihr Gegenteil verkehren, wenn der Makrokosmos auf ihn Einfluss nimmt oder mit ihm in Berührung kommt. Denn im empfindsamen Roman gilt die Eintracht der Liebenden in der Natur zunächst als Skizzierung einer Gesellschaftsutopie, die allerdings deren Verletzung unnachsichtig bestraft. ${ }^{15}$ In Saint-Pierres Roman Paul et Virginie ist die Natur zugleich Segen und Fluch für das Liebespaar. Da Virginie die Insel verlassen hat, wird ihr die Rückkehr ins diskursabstinente Paradies versagt. Saint-Pierre greift somit auf den alttestamentarischen Schöpfungsmythos vom Verlust der Unschuld durch die Erkenntnis zurück. Ich will nun versuchen, diese Bewertungen und Inszenierungsformen der Raum-, Sozial- und Lebensutopie ,Inselexistenz' nachzuzeichnen und in STORMS Realismus eine skeptische

14 Eine einführende Werkbiographie zu StORM, mit originellen Affinitäten zur psychoanalytischen Interpretation, findet sich in der Monographie von REGINA FASOLD (1997).

15 Dazu schreibt Urs Bitterli: „Nachdem der Schriftsteller [das ist Bernardin de Saint-Pierre, T.V.] nach Paris zurückgekehrt war und dort Jean-Jacques Rousseau getroffen hatte, entwarfer, nunmehr aus der Ferne urteilend, in seinem Roman Paul et Virginie ein zauberhaft-entrücktes Bild der Insel; es ist dies vielleicht das erste literarische Produkt, in dem das Exotische nicht mehr bloß als Akzent und Kontrast auftritt, sondern sich in einem ständig präsenten künstlerischen Stilwillen manifestiert"(BITTERLI 1987:23). 
Betrachtung ontologischer oder anthropologischer Konzepte wie ,Raum‘ herauszuarbeiten, die ihn doch von RAABE und STIFTER unterscheidet.

Das Gemälde in Richards Salon, der junge Förster und Richards Angstträume oder auch die Gespräche mit Franziska selbst evozieren permanent Zweifel in dem alternden Mann. Sie lassen das Ansinnen Richards während des gesamten Plots durchweg als hoffnungslos erscheinen und machen sein emsiges und beharrendes Bemühen dadurch auch tragisch. Das hat in der Tat, wie es auch die junge Baronin Hermione kritisch bemerkte, nichts mehr mit Melancholie zu tun, denn diese ermöglicht immer noch ein stimmungsvolles Sich-selbstGefallen im Schmerz, sondern ist in der Tat pessimistisch. Insofern ist die Paarkonstellation bei STORM auch kein literarisches Klischee, sondern nur ein typisches Beziehungsgeflecht im Interesse der Darstellung gescheiterter Lebensentwürfe. STORM selbst hat übrigens in den Vorarbeiten zum Waldwinkel erheblich an der Schlussepisode gefeilt, um nicht ins Triviale abzugleiten. ${ }^{16}$ Auch versuchte er in den Briefen an die junge Baronin das von ihm gewählte Sujet zu rechtfertigen, indem er auf eine reale Begebenheit verwies, die ihm in seiner Tätigkeit als Amtsrichter zugestoßen war. Letzteres ist zwar im Kontext der Realismusdebatte nicht uninteressant - nach dem Motto: Die Novelle erzählt von typisch menschlichen Schicksalen und rekurriert positivistisch auf Aktenmaterial - jedoch als Rechtfertigungsstrategie unnötig. ${ }^{17}$ Dass STORM ein Vertreter des Zweifels ist und jedes Sinnkonzept eigentlich relativiert, wird bereits in der frühen Lyrik deutlich. ${ }^{18}$ Der Wunsch nach praktischer Realisierung in der späteren Novelle von 1874 enthält dieselbe Crux wie all die Reisen zu den südlichen Gestaden bei den französischen Spätaufklärern und Frühromantikern. So bemerkt auch Wuthenow, „daß die Reisen [...] die zuvor noch das utopische Denken genährt hatten, nunmehr nur noch dazu beitragen, die Utopie zu zerstören“" (WUTHENOW 1985:334). Diese an Ernst Blochs Utopiebegriff geschulte und unausweichliche Dilemma-Dialektik resultiert aus dem Wunsch nach Umsetzung

16 Wahrscheinlich mit Blick auf ADALBERT STIFTERS versöhnlicher endende Novelle Die Narrenburg, die übrigens als utopischer Ort von den beiden Protagonisten gemeinsam, also ohne Zwang errichtet wird, nannte STORM seinen Text zunächst Im Narrenkasten. Doch bereits das Attribut des Kastens, welches an die Stelle des romantischen Motivs der Burg tritt, lässt unweigerlich einen Zustand der Enge und des Eingesperrtseins assoziieren.

17 Der Brief findet sich in STORM (1987:865f.). Vgl. auch den späteren Brief an Ada Christen vom 19.Oktober 1876 (STORM 1987:866).

18 Das verbindet ihn wohl eher mit resignativen Autoren wie Ferdinand von Saar als mit STIFTER oder RAABE. 
des Insel-Konzepts in die eigene Lebenspraxis, also in einen ganz und gar nicht insularischen Diskurszusammenhang, und scheitert daher. Denn das Reisen zur Insel ist eine ähnliche Umsetzung wie das verzweifelte Errichten einer solchen. In der zu diskutierenden Novelle von 1874 ist daher eine solche Hoffnungslosigkeit nicht nur das eigentliche Thema, sondern auch die charakteristische Stimmung, die sich, beim Lesen immer wieder bemerkbar, in Abständen durch den Text zieht, angefangen von den obskuren Bekleidungsszenen, die sich zwischen Richard und Franziska abspielen - er stattet sie regelrecht aus und formt sie nach seiner Imagination zurecht ${ }^{19}$ - bis hin zu dem Motiv des Gemäldes in Richards Arbeitszimmer:

Es war eine weite Heidelandschaft, vielleicht die an dem Waldwinkel selbst gelegene, hinter welcher eben der erste rote Sonnenduft heraufstieg; in der Ferne sah man, gleich Schattenbildern, zwei jugendliche Gestalten, eine weibliche und eine männliche, die Arm in Arm, wie schwebend, gegen den Morgenschein hinausgingen; ihnen nachblickend auf einen Stab gelehnt, stand im Vordergrunde die gebrochene Gestalt eines alten Mannes. Als Richard jetzt von dem Bilde auf die Umrahmung desselben hinüberblickte, trat ihm dort, halb versteckt zwischen allerlei Arabesken, eine Schrift entgegen, die bei näherem Anschauen in phantastischen Buchstaben um das ganze Bild herumlief. Dein jung' Genoß in Pflichten / Nach dir den Schritt tät' richten; / Da kam ein and'rer junger Schritt, / Nahm deinen jung' Genossen mit; / Sie wandern nach dem Glücke, / sie schau'n nicht mehr zurücke. So lauteten die Worte. Lange stand Richard vor dem Bilde, das er früher kaum beachtet hatte. Würde das Antlitz jenes einsamen Alten, wenn es sich plötzlich zu ihm wendete, die Züge des Erbauers dieser Räume zeigen, oder war diese Gestalt das Alter selbst, und würde sie - nur eines vermessenen Worts bedurfte es vielleicht - sein eigenes Antlitz ihm zukehren? - Wehte nicht schon ein gespenstisch kalter Hauch von dem Bilde zu ihm herab? (STORM 1987:254f.)

Das Gemälde bildet eine fast schon emblematische Einheit mit Versen, welche die Situation des Verlassenen noch verstärken, indem sich beide Medien gegenseitig kommentieren. Richard betreibt eine verhängnisvolle und selbstidentifikatorische Allegorese der emblematischen Darstellung und bringt sie mit dem Alter an sich in Verbindung. Zugleich enthält seine Ekphrasis zahlreiche Oppositionspaare, die nicht nur für sein Verhältnis zu Franziska, sondern auch für die

Die auf diese Weise erfolgte Verkünstlichung / Ästhetisierung der Frau nach der Vorstellung des männlichen Künstlers ist nicht nur aus gendertheoretischer Perspektive von Bedeutung, sondern auch für den Ästhetizismus der Décadence. Die Verdrängung der Realität über - fast schon fetischisierte - Kleidung beherrscht auch das Liebeskonzept des Dandys und Katholiken Hugo in Georges Rodenbachs Novelle Bruges - la - morte / Das tote Brügge (1892) und in Erich Korngolds Opernvertonung Die tote Stadt von 1920. 
Beziehung der Insel zur Umwelt charakteristisch sind. Der Alte und das Alter selbst werden zur insularen Existenz, während das junge Paar in die Welt hinaus schreitet. Auf diese wartet der Morgenschein als ein Zeichen des Aufbruchs und des Neubeginns, von dem der Alte und Zurückbleibende in seiner insularischen Existenz getrennt ist. Der Morgenschein weckt hier eindeutig die jüngere Generation, während Richard quasi schlafend seinem Kokon verhaftet bleibt.

Da das Paar keinen einzigen Rückblick mehr auf den Alten riskiert, sind die Dichotomien zwischen zwei Generationen, Beziehungskonzepten und auch Raumvorstellungen absolut manifest geworden. Die Entfernung von der Insel impliziert Dynamik, das Verharren in ihr den Stillstand, mit welchem der Betrachter sein eigenes Alter als einen Zustand der Abgeschiedenheit vom Leben erkennt, was jegliche Glücksvorstellung der Insularität aushebelt, da es zudem noch Richards totale Isolation als den eigentlichen Inselzustand prognostiziert, ihn zur radikalen figurativen Realisierung des Narrativs ,Waldwinkel' werden lässt. Das Altern wird damit als absolut allein zu erduldender Prozess begriffen, der nicht geteilt werden kann, vor allem nicht als ein Generationen und wohl auch Geschlechter übergreifendes Modell. Hier ist STORMS Pessimismus unüberbietbar. Männer wie Richard „haben den Heilsweg verfehlt, den die von christlicher Heilslehre weitgehend bereinigte bürgerliche Wertewelt den Menschen noch offen lässt: die Erfüllung des Lebens durch eine sinnstiftende Heirat und die Gründung einer glücklichen Familie“ (KÜNG 2015:23f.). ${ }^{20}$ Das besagte Bild nimmt Richards Schicksal in unheimlicher Weise vorweg:

Er wußte vorher schon, was er finden würde. Was ihm gehörte, lag unberührt; das Päckchen mit Franziskas Namen war verschwunden. - Eine Weile suchte er noch nach einem Zettelchen von ihrer Hand, einem Wort des Abschieds oder was es immer sei; er räumte das ganze Fach aus, aber es fand sich nichts. - Durch die Fenster brach der erste Morgenschein und ließ das alte Türbild aus der Dämmerung hervortreten. Als er zufällig den Blick dahin warf, überkam ihn ein wunderlicher Sinnentrug; der einsame Alte dort am Wege hatte ja den Kopf gewandt und sah ihn an. Die Sonne stieg höher, an den Treppen leuchteten die Blumen der Vergessenheit. Richard hatte die Augen noch immer nach dem Bilde. Es war sein eigenes Angesicht, in das er blickte. (STORм 1987:276f.)

Diese Transformation ist jedoch nicht so sehr als eine Reminiszenz STORMS gegenüber romantischer Phantastik zu verstehen, sondern als Bestätigung der ahnungsvollen Antizipation des Scheiterns des Waldwinkel-Konzepts. Richard wird Teil des Bildes und damit seiner eigenen Vorstellung vom Insel-Zustand,

Letzteres ist auch nicht mehr auf einer Insel möglich. 
der jedoch von Anbeginn nicht auf Gemeinsamkeit, sondern auf Zwangsverhältnisse und Tauschprozesse gegründet war. Dass ihm Franziska keinerlei Abschiedsworte hinterließ, dokumentiert quasi als Nicht-Dokument das Nichtvorhandensein eines gemeinsamen sozial- und liebesutopischen Konzepts. Das Ausbleiben jeglicher Erklärung kommuniziert auch die sprachliche Isolation Richards. Das antizipatorische Procedere der Bildallegorese findet sich bereits in STORMS Novelle Eine Malerarbeit (1867) angedeutet. Allerdings ist es dem verkrüppelten Maler Edde Brunken möglich, das Schicksal gegenüber dem (von ihm selbst geschaffenen) Bild, welches ihn als verlassenen und verlachten Trottel diffamiert, aktiv zu verändern und sein Leben aus dem Holze zu schnitzen, welches ihm zur Verfügung steht, wie die etwas simpel anmutende Moral von der Geschicht' nun einmal lautet. Trotz aller Phantasie ist Richard kein Künstler und zugleich auch kein Realist. Er bleibt in seiner Vision vom Liebesglück im Waldwinkel gefangen und kann sein Schicksal nicht aktiv mitgestalten. Im Gegenteil: Das Gemälde, welches einen verlassenen alten Mann zeigt, der einem jungen Liebespaar hinterherblickt, wird zur deterministischen Vision oder zum Praesagium seiner späteren Lebenssituation, an welcher der Möchtegerninsulaner nichts ändern kann, da ihm überhaupt Aktivismus sehr fremd zu sein scheint. Das separiert ihn auch von den pädagogisch-romantischen Konzepten der Wald-Insel-Existenz, wie sie beispielsweise noch für Henry David Thoreaus Walden (1859) spezifisch war, worin der Wald symbolisch für einen Ort der Läuterung und der Rückkehr zum Wesentlichen der menschlichen Existenz in Anspruch genommen werden konnte, ähnlich wie bei Bernardin Saint-Pierres ,indischer Hütte'. Von diesen Wesentlichkeiten ist Richard bei STORM jedoch sehr weit entfernt.

Der Altersunterschied und der Interessenkonflikt zwischen ihm und Franziska lassen keine andere Realität zu. Das Haus im Waldwinkel steht nicht für ein dauerhaftes Glück. Dabei hätte er spätestens seit Bernardin de Saint-Pierres Rousseau-Roman Paul et Virginie wissen müssen, dass der Mikrokosmos der Insel zusammenbricht, wenn der Makrokosmos auf ihn Einfluss nimmt oder mit ihm in Berührung kommt. ${ }^{21}$ Letzteres geschieht in STORMS Waldwinkel

21 Obgleich sogar in historischen Reiseberichten wie der berühmten Voyage autour du monde (1766-1769) von Bougainville aus dem 18. Jahrhundert das insulare Prinzip partiell und nur für einen kurzen Zeitraum erfahren werden kann. Das gelingt jedoch nur, weil sich die Schiffsmannschaften den herrschenden Sitten Tahitis anpassen und in amourösen Verhältnissen mit diversen Inselschönheiten ihre christlichen und europäischen Moralvorstellungen über Bord werfen. Da die Reise als Aktion 
nicht nur in Gestalt des jungen Försters, der Franziska mit der Außenwelt konfrontiert und sie erkennen lässt, dass sie von Alter, Interessen und Persönlichkeitsstruktur einem anderen Soziotop zugehörig ist. Auch im Waldwinkel selbst dominieren erotische Obsessionen und wirtschaftlich-soziale Interessen die Motivation seiner Einwohner*innen. Richards Bestreben, Franziska nicht nur habituell, sondern auch vestimentär an seine Lebenswelt anzupassen sowie sie über Wertpapiere finanziell an sich zu binden, sind jedem rousseauistischen Konzept entgegengesetzte Tauschbeziehungen. Gerade das wirtschaftliche Argument bringt Franziska dazu, sich die Papiere anzueignen und den insularen Raum zu verlassen, da letzteres selbst Teil der externen Kapitalgesellschaft ist. Das Außen ist quasi schon bei der geistig-konstruktiven Grundsteinlegung des Domizils unbestrittener Bestandteil des Innen. In beiden Fällen gestaltet jedoch der Raum als übergeordnetes Narrativ die untergeordneten Narrationen der Figuren und stellt die dominierende Diegesis dar. Gerade die fatalen Auswirkungen des Scheiterns der Raumvorstellung auf alle weitere Figuren- und Plotentwicklung dokumentieren das.

\section{Auswertung}

Da die Sozialform der Insel auf der Beziehung mit Franziska beruhte, bricht sie nach ihrem Abgang für Richard zusammen, unbarmherzig veranschaulicht durch den toten Hund, welcher die Isolation des Alten noch einmal verstärkt. ${ }^{22}$ Vielleicht sind all das auch Klischeefiguren, aber Klischees sind oft auch als übertriebene Zuspitzungen der Realität zu werten und dienen hier dazu, die Tragik des Scheiterns besonders plastisch (oder: überdeutlich) zu visualisieren. Insofern wird STORM auch hier seinem in der späteren Novellentheorie Eine zurückgezogene Vorrede (1881) geäußerten Anliegen gerecht, für die menschliche

und Narrativ jedoch fortgesetzt wird, hat die Crew vom Paradies nur gekostet, was den utopischen Charakter und die Irrealität des Ortes wieder bekräftigt. Die diskursive Prägung, die sich in der Fortsetzung der Fahrt symbolisch kundtut, verhindert die Aufrechterhaltung des Fluchtraums als dauerhaftes Habitat (vgl. DE BougAINVILLE 1980). In Storms Novelle von 1874 legt keiner der Waldinsel-Besucher*innen bzw. Bewohner*innen die diskursive Ummantelung ab. Sie ist der Insel inskribiert, die Glücksgefährdung droht von innen, wie von DE CORT (vgl. 1971) bemerkt.

Umso stärker und zugleich absurder wirken im Vergleich dazu die Glücksmomente Richards, wenn er von einer gemeinsamen Zukunft mit Franziska träumt, oder sie nach seinem eigenen Geschmack (und gemäß seiner Erinnerung) vestimentär ausstaffiert (vgl. unter anderem STORM 1987:240). 
Existenz relevante und diese auch mitbestimmende Situationen zu beschreiben, selbst wenn es eine anthropologische Konstante ex negativo ist, wie das Alleinsein und die damit verbundene Absage an rousseauistische Utopien, die mit Blick auf eine gescheiterte Insel-Existenz durch Rainer Maria Rilkes Gedicht Der Blinde (1908) besonders luzide visualisiert wurde: „Nichts ist mehr mit mir verbunden. / Ich bin von allem verlassen. - Ich bin eine Insel“" (RILKE 1980:346f.). Das reicht nun nicht mehr für das auf Gemeinsamkeit (auch mit Blick auf die Konstruktion des utopischen Raums) beruhende Konzept von STIFTERS Narrenburg. Das reicht nur noch für den selbst zur Insel, also zum isolierten Raum und wohl auch zum Narrativ werdenden Hagestolz (1844), den der Dichter erkennen lässt: „Er saß ganz einsam auf seiner Insel; denn wie er einmal selber gesagt hatte, es war alles, alles zu spät, und was versäumt war, war nicht nachzuholen“ (STIFTER 1975a:909). Das Zurückbleiben auf der Insel wird - und hier differenziert STIFTER zwischen Zeit und Stagnation - auch als ein Herausfallen aus der Zeit für den Insulaner gedeutet, weil „seine Sprossen nicht mit hinunter gehen in dem Strome der Zeit" (STIFTER 1975a:910). Damit nimmt auch STIFTER diesem Inselprojekt alle utopische Qualität, denn der nur aus sich selbst heraus lebende Insulaner wird als quasi unfruchtbares Produkt gebrandmarkt, „weil an ihm schon alles im Sinken begriffen ist“" (STIFTER 1975a:910). STORMS Blick verhindert ebenso die Reanimation romantischer Waldeinsamkeit. Letztere bildet einen schon längst vergangenen Horizont, an dem die Unmöglichkeit einer Realisierung gemessen wird.

\section{Literatur}

Bitterli, Urs (1987): Die exotische Insel. In: Koebner, Thomas / Pickerodt, Gerhard (eds.): Die andere Welt. Studien zum Exotismus. Frankfurt a. M., 11-30.

Bollenbeck, GeORg (1988): Theodor Storm. Eine Biographie. Frankfurt a. M.

Brasillach, Robert (1953): Ein Leben lang. Roman in sechs Episoden. Aus dem Französischen von Gertrud Grote. München.

Broser, PATRICIA (2012): Insel. In: Butzer, GÜNTER / JACOB, JOACHIM (eds.): Metzler Lexikon literarischer Symbole. Stuttgart / Weimar, $199 \mathrm{f}$.

BRUNNER, HORST (1967): Die poetische Insel. Inseln und Inselvorstellungen in der deutschen Literatur. Stuttgart.

De Bougainville, Louis-Antoine (1980): Reise um die Welt, ed. PopP, Klaus-GeOrG. Berlin.

De Chateaubriand, Francois René (1962): René-Atala. Aus dem Französischen von Trude Geissler. Stuttgart. 
DE CORT, JoSEF (1971): Das Raumgefüge als Gestaltungselement in der epischen Dichtung. In: Archiv für das Studium der neueren Sprachen und Literaturen 207:321-340.

DETERING, HEINRICH (1984): Apokalyptische Bedeutungsstrukturen in Raabes Das Odfeld. In: Jahrbuch der Raabe-Gesellschaft, 87-98.

FASOLD, REgINA (1997): Theodor Storm. Stuttgart / Weimar.

FRANK, MichAEL C. (2012): Sphären, Grenzen und Kontaktzonen. Jurij Lotmans räumliche Kultursemiotik am Beispiel von Rudyard Kiplings Plain Tales from the Hills. In: Frank, Susi K. / RuHE, CORNELIA / Schmitz, AlEXANDER (eds.): Explosion und Peripherie. Jurij Lotmans Semiotik der kulturellen Dynamik revisited. Bielefeld, 217-246.

HAAG, SASKIA (2012): Auf wandelbarem Grund. Haus und Literatur im 19. Jahrhundert. Freiburg im Breisgau / Berlin / Wien.

Hallet, Wolfgang / Neumann, Birgit (2009) (eds.): Raum und Bewegung in der Literatur. Die Literaturwissenschaftenund der Spatial Turn. Bielefeld.

Killy, Walter (1963): Wilhelm Raabe: Das Odfeld. In: von Wiese, Benno (ed.): Der deutsche Roman. Vom Barock bis zur Gegenwart. Struktur und Geschichte, Bd. II. Düsseldorf, 128-145.

KÜNG, Peter (2015): Die Krise der liberalen Anthropologie in der Literatur des Bürgerlichen Realismus. Männlichkeit, Bürgerlichkeit und Individualität bei Theodor Storm, Theodor Fontane und Paul Heyse. Würzburg.

LAAGE, KARL ERnSt (1987): Kommentar. In: LAAGE, KARL ERnST / LOHMEIER, DiETER (eds.): Theodor Storm. Sämtliche Werke in vier Bänden, Bd. 2. Frankfurt a. M., 867f.

Lotman, Juriu (1974): Das Problem des künstlerischen Raums in Gogols Prosa. In: EIMERMACHER, KARL (ed.): Aufsätze zur Theorie und Methodologie der Literatur und Kunst. Aus dem Russischen von Karl Eimermacher. Kronberg im Taunus, 200-271.

Maler, Anselm (1978): Exotische Hütten. Im Paradies des Populärromans zwischen Restauration und Revolution. In: UEDING, GERT (ed.): Literatur ist Utopie. Frankfurt a. M., 189-220.

Martínez, Matías / Scheffel, Michael (2016): Einführung in die Erzähltheorie. München.

Müller-TAmm, JutTa (2007): ,Alles nicht zu Ende. Alles falsch... ‘. Allegorie und Erzählstruktur in Stifters Narrenburg. In: Zeitschrift für Germanistik N.F. 17:561-574.

OpPermann, Hans (1967): Der passive Held. Raabe: Das Odfeld. In: Jahrbuch der Raabe-Gesellschaft, 31-50.

RaAbe, Wilhelm (1985): Das Odfeld. In: Schrader, HANS-JÜrGen (ed.): Wilhelm Raabe. Werke in Einzelausgaben, Bd. 6. Frankfurt a. M.

Rilke, RaIner Maria (1980): Der Blinde. In: Rilke-ArchiV IN VERBINDUNG Mit SiEBER-RILKe, Ruth (eds.): Rainer Maria Rilke. Werke in sechs Bänden, Bd. II. Frankfurt a. M., 346f.

RitTERSON, Michael (1976): Rückwendung, Vorausdeutung und Erzählablauf in Wilhelm Raabes Das Odfeld und Hastenbeck. In: Jahrbuch der Raabe-Gesellschaft, 107-132. 
SCHLEGEL, FRIEDRICH (1988): Geschichte der alten und neuen Literatur, Vorlesungen, gehalten zu Wien im Jahre 1812. In: BEHLER, ERNST / EICHNER, HANS (eds.): Kritische Schriften und Fragmente. Studienausgabe in sechs Bänden, Bd. 4. Paderborn / München / Wien / Zürich, 1-234.

SCHRÖDER, HANS (1985): Der Raum als Einbildungskraft des Dichters bei Stifter. Frankfurt a. M.

StifTer, AdAlbert (1975): Die Narrenburg. In: ders.: Studien. Vollständige Ausgabe nach dem Text der zweiten Auflage (1847-1850). München, 277-380.

StifTer, AdAlbert (1975a): Der Hagestolz. In: ders.: Studien. Vollständige Ausgabe nach dem Text der zweiten Auflage (1847-1850). München, 793-910.

StOrm, THEOdOR (1987): Waldwinkel. In: LAAGE, KARL ERnST / LOHMEIER, DiETER (eds.): Theodor Storm. Sämtliche Werke in vier Bänden, Bd. 2. Frankfurt a. M., 221-279.

TurgenJew, IwAN (1968): Briefe. Aus dem Russischen von Günter Dalitz, aus dem Französischen von Irene Zimdahl, aus dem Englischen von Friedrich Baadke, ed. SCHultze, Christiane. Berlin / Weimar.

VOß, TORSTEN (2010): Der andere Storm. Temporäre Negativität als Sprache radikaler Aufrichtigkeit. Eine ästhetisch-anthropologische Perspektive. In: Schriftenreihe der Theodor-Storm-Gesellschaft 59:81-96.

WUTHENOW, RALPH RAINER (1985): Inselglück. Reise und Utopie in der Literatur des XVIII. Jahrhunderts. In: VOßKAMP, WILHELM (ed.): Utopieforschung. Interdisziplinäre Studien zur neuzeitlichen Utopie, Bd. 2. Frankfurt a. M., 320-335.

WÜNSCH, MARIANNE (1999): ,Tod' in der Erzählliteratur des deutschen Realismus. In: Jahrbuch der Raabe- Gesellschaft, 1-14. 